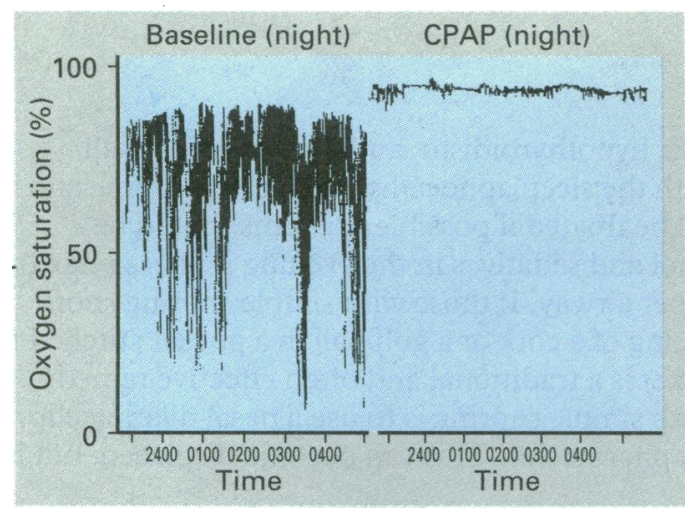

Overnight oxygen saturation traces in a patient with the sleep apnoea/hypopnoea syndrome. Left: Baseline tracing when the patient was receiving no treatment. Right: Tracing taken the next night during treatment with CPAP, showing abolition of episodes of desaturation by normalisation of the breathing pattern.
This often results from an air leak that develops through the mouth when the patient's muscle tone drops during sleep and can often be helped by the addition of a chin strap. If this fails intranasal steroids or atropinic drugs are often helpful. It is occasionally necessary to add a humidifier to the system.

The main drawback to CPAP is that patients need to use the machine every night of their lives, although this is a small price to pay for the benefit obtained. In practice most patients use the machine for about four hours a night, some use it for part of the night, and some occasionally omit it altogether.

Fewer than $1 \%$ of patients with sleep apnoea have apnoeas that do not respond to CPAP and result from cessation of respiratory effort-true central sleep apnoeas. Most of these have coexisting neurological diseases and are best treated either by intermittent positive pressure ventilation through a nasal mask or by diaphragmatic pacing.

\title{
Conclusion
}
Always ask:
- Do you fall asleep each day when not in bed?
- Do you snore loudly and intermittently?

The main problem in the medical management of the sleep apnoea/hypopnoea syndrome is underawareness of the condition. Clinicians must bear the possibility of the diagnosis in mind and ask the appropriate questions routinely.

N J Douglas is a reader, respiratory medicine unit (RIE), University of Edinburgh and director, Scottish National Sleep Laboratory.

The ABC of Sleep Disorders has been edited by Colin M Shapiro, professor of psychiatry, University of Toronto, Canada.
Foreign bodies in the splinters, do not always show on radiographs

Birmingham and Midland Eye Hospital, Birmingham B3 2NS

Andrena M McElvanney, senior house officer Alistair R Fielder, professor of ophthalmology

Correspondence to: Miss McElvanney.

Lesson of the Week

\section{Intraocular foreign body missed by radiography}

\author{
Andrena M McElvanney, Alistair R Fielder
}

Foreign bodies entering the eye may give rise to a wide range of complications including hyphaema, cataract, vitreous haemorrhage, and retinal tears and detachment. ${ }^{1-3}$ Inflammation and infection may also affect the whole ocular cavity, leading to endophthalmitis. ${ }^{4}$

Certain metallic foreign bodies within the eye may produce retinotoxic ions. Ferrous ions destroy retinal photoreceptors and pigment epithelial cells, and copper induces siderosis and chalcosis. Thus, intraocular foreign bodies should be removed promptly to prevent these reactions and minimise intraocular inflammation and infection, which commonly causes a destructive fibrovascular response that may ultimately result in blindness. ${ }^{46}$

\section{Case report}

A 42 year old man presented to our accident and emergency department complaining of a gritty sensation in his left eye. Two days earlier he had been fitting a metal tool on to a lathe when he felt a sudden sharp sensation in his left eye. He had not been wearing safety goggles. His left eye became irritable and was irrigated with normal saline by the occupational health nurse.

The following day, as his eye was still troublesome, he attended a general accident and emergency depart- ment, where the eye was examined under a hand held light and then stained with fluorescein. No abnormality was seen and orbital radiographs showed normal results. Chloramphenicol ointment was applied to the eye overnight, and the patient was advised to seek an ophthalmic opinion the following morning, although he was not formally referred.

On presentation to the ophthalmic department visual acuity (without correction) was $6 / 5$ in the right eye and $6 / 6+2$ in the left. Examination of the left eye under a slit lamp showed injected conjunctiva, a small self sealed perforation of the cornea, and a metallic foreign body on the surface of the iris (fig 1). Repeat orbital radiographs were taken, and, again, showed no radio-opaque foreign body. An intraocular foreign body was, however, detected on ultrasound scanning of the globe (fig 2) and was surgically removed with good result.

\section{Discussion}

In many accident and emergency departments it is standard practice to perform orbital radiography in patients with ocular trauma resulting from high velocity particles. The need for such radiological investigation, however, has been debated. The detection of a radioopaque foreign body depends on its relative density 


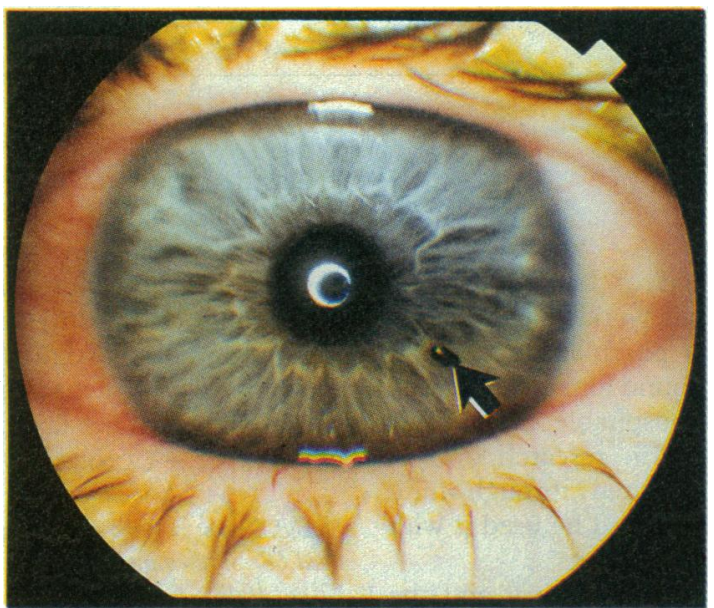

FIG 1-Iris with metallic splinter (arrow)

compared with water. ${ }^{7}$ Bray and Griffiths reviewed the indications for and the results of radiography performed in their eye department over five years. ${ }^{8}$ Their results suggested that most orbital radiography was needless. Although they did not discuss the criteria influencing the detection of foreign bodies, they reported a detection rate of $90 \%$ for metallic splinters. ${ }^{8}$

In a prospective study of ultrasonography in the assessment of foreign bodies in pigs' eyes Bryden et al reported a detection rate of $69 \%$ for metallic splinters. ${ }^{9}$ Failure in detection did not relate to size: most of the larger foreign bodies were apparent on scanning, but not only the smallest escaped detection. ${ }^{9}$ (In this study the composition of the metallic foreign bodies was not defined.) Radiography is even less reliable in detecting glass, perspex, or wood. ${ }^{7}$

In our patient a metallic intraocular foreign body was visible clinically on the iris but was not detected in radiographs taken on separate occasions. An ultrasound

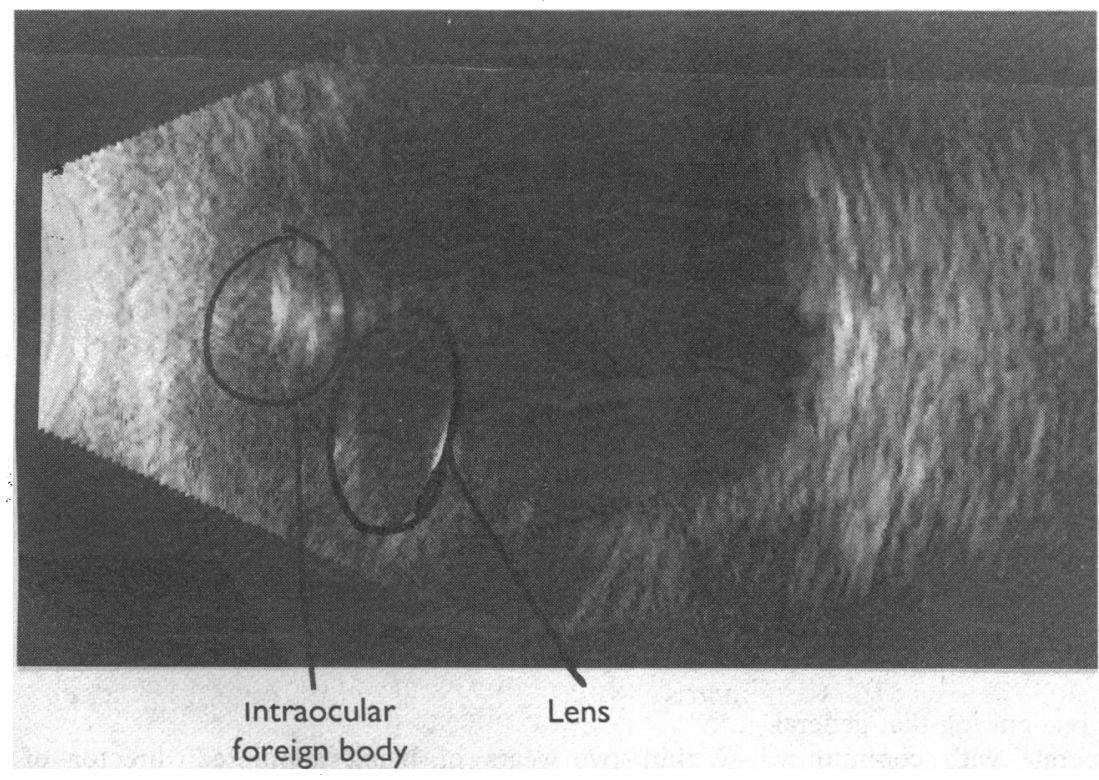

FIG 2-Ultrasound scan of left eye showing intraocular foreign body with acoustic echo anterior to lens scan of the left eye, however, showed the foreign body within the anterior segment. Bryden et al established an overall detection rate for metallic and non-metallic intraocular foreign bodies of $93 \%$ for ultrasonography and $40 \%$ for plain radiography. ${ }^{9}$

Our case report highlights the limited reliability of orbital radiography in detecting an intraocular foreign body and the need for adequate clinical examination of the eye. It also suggests that some radiological investigations in patients may be inappropriate. Bray and Griffiths stated that when an intraocular foreign body was visible on radiography, the eye always showed concomitant evidence of penetration. They suggested that orbital radiography should be performed in patients exposed to high velocity particles and in those with clinical evidence or strong suspicion of ocular or orbital trauma, including subconjunctival haemorrhage. A senior opinion should be sought when an intraocular foreign body cannot be excluded by junior staff. This would help to reduce both the unnecessary exposure of patients to ionising radiation and the high cost of such investigations. ${ }^{8}$

Computed tomography may also be used, but it is expensive and not always readily available. ${ }^{10}$ Ocular ultrasonography is comparatively inexpensive, acceptable to patients, and sensitive. However, it requires an experienced operator and reporter and is not a suitable option for a busy accident and emergency department. Furthermore, specific frequencies $(10 \mathrm{MHz})$ and sensitivities of less than 50 decibels are required, necessitating that equipment be dedicated to ophthalmic use.

Appropriate ocular assessment is mandatory if an intraocular foreign body is suspected. This must include examination for a possible entry site on the eyelids, conjunctiva (haemorrhage and chemosis), and cornea. In addition, the iris should be scrutinised for irregularity, which may indicate that a foreign body is impacted within it. Lens opacity should be noted, as cataract formation may result from the passage of a foreign body through the eye. Finally, the posterior segment must be examined for the foreign body, haemorrhage, or retinal detachment. Thus radiography is useful as an adjunct to clinical examination but normal radiographs do not exclude the possibility of an intraocular foreign body.

1 Williams DF, Mieler WF, Abrams GW, Lewis H. Results and prognostic factors in penetrating ocular injuries with retained intraocular foreign bodies. Ophthalmology 1988;95:911-6.

2 Williams DF, Mieler WF, Abrams GW. Intraocular foreign bodies in young people. Retina 1990;10:S45-9.

3 Shock JP, Adams DA. Long-term visual acuity results after penetrating and perforating ocular injuries. Am $\mathcal{f}$ Ophthalmol 1985;100:714-8.

4 Mieler WF, Ellis MK, Williams DF, Han DP. Retained intraocular foreign bodies and endophthalmitis. Ophthalmology 1990;97:1532-8.

5 Benson WE. Intraocular foreign bodies. In: Tasman W, Jaeger EA, eds. Duane's clinical ophthalmology. Vol 5. Philadelphia: Lippincott, 1991:1-15.

6 Percival SPB. A decade of intradcular foreign bodies. Br f Ophthalmol 1972;56:454-61.

7 De Lacey G, Evans R, Sandin B. Penetrating injuries: how easy is it to see glass (and plastic) on radiographs? Br $\mathcal{\text { R Radiol } 1 9 8 5 ; 5 8 : 2 7 - 3 0}$

8 Bray LC, Griffiths PG. The value of plain radiography in suspected intraocula foreign body. Eye 1991;5:751-4.

9 Bryden FM, Pyott AA, Bailey M, McGhee CNJ. Real time ultrasound in the assessment of intraocular foreign bodies. Eye 1990;4:727-31.

10 Zinreich MD, Miller NR, Aguayo JB, Quinn C, Hadfield R, Rosenbaum AE Computed tomographic three-dimensional localization and compositional evaluation of intraocular and orbital foreign bodies. Arch Ophthalmol 1986; 104:1477-82.

(Accepted 20 January 1993) 